\title{
Rendimento do processamento dos produtos oriundos da fibra vegetal da piaçava (Attalea funifera)
}

\author{
Noara Modesto PIMENTEL ${ }^{1 *}$, Claudio Henrique Soares DEL MENEZZI ${ }^{1}$ \\ ${ }^{1}$ Departamento de Engenharia Florestal, Universidade de Brasília, Brasília, DF, Brasil. (ORCID: *; 0000-0003-3369-2392) \\ *E-mail: noarapimentel@gmail.com (ORCID: 0000-0003-4775-7986)
}

Recebido em 21/03/2019; Aceito em 27/06/2019; Publicado em 04/02/2020.

\begin{abstract}
RESUMO: O presente trabalho abordou uma análise integrada do processamento dos Produtos Florestais Não Madeireiros (PFNM) oriundos da fibra vegetal junto aos diversos atores da cadeia produtiva da piaçava da Bahia (Attalea funifera Mart. Arecaceae). Atualmente a renda média do agroextrativista é aproximadamente $\mathrm{R} \$$ $600,00 /$ mês no sistema convencional. Neste caso as fibras vegetais são processadas e comercializadas nas formas: bruta, limpa, cortada, fino-média e grosso-média. Para obtenção de uma análise integral da cadeia produtiva, a metodologia empregada consistiu em: ensaios de rendimento em seis sistemas produtivos diferentes e entrevistas com gestores, diálogos com produtores e agroextrativistas tradicionais. Os resultados do processamento da fibra vegetal apontaram que 47,3\% de resíduos são descartados, 35,6\% são fibras finomédia, 5,3\% grosso-média e 17\% de "fita" são aproveitadas. Os ganhos do agroextrativista, supondo um cenário em que todas as etapas do processo produtivo de confecção das vassouras de piaçava fossem realizadas, corresponderiam a uma renda mensal líquida de $\mathrm{R} \$ 1.664,63$, muito acima da renda média atual.
\end{abstract}

Palavras-chave: fibras naturais; produtos florestais não madeireiros (PFNMs); piaçava da Bahia; agroextrativismo; Mata Atlântica; resíduos florestais.

\section{Yield of the products processing from the vegetable fiber of piassava palm tree (Attalea funifera)}

\begin{abstract}
This article point out an integrated analysis of the processing of non-timber forest products (NTFPs) originating from the vegetable fiber with the different actors of the productive chain of the piassava palm tree of Bahia (Attalea funifera Mart. Arecaceae). Currently the average income of the of the agroextractivist is approximately US $\$ 256,13$ per month in the conventional system. In that case, the vegetable fibers are processed and commercialized in the forms: raw fiber, clean fiber, cut fiber, thin-medium fiber and thickmedium fiber. In order to obtain complete analysis of piassava palm tree production chain, the methodology used consisted of: performance tests in six different production systems, the interviews with managers and dialogues with traditional producers and agroextractivists. The results of the processing of the vegetable fiber indicated that $47,3 \%$ of the residues are discarded, 35,6\% are thin-medium fibers, 5,3\% thick-medium fibers and 17\% of "stripes" (by-product) are used. The gains of the agroextractivist, supposing scenario in which all stages of the production process of making the piassava brooms were held, would be followed, might correspond to a monthly net reve nue of US\$710,59 well above the current average yield.
\end{abstract}

Keywords: natural fibers; Non-timber forest products (NTFPs); Bahia piassava; Atlantic forest; Exploitation of forest residues.

\section{INTRODUÇÃO}

A piaçava da Bahia (Attalea funifera Mart. Arecaceae) é uma palmeira endêmica da restinga (ecossistema associado ao Bioma Mata Atlântica), que fornece uma fibra vegetal resistente, frutos e folhas em abundância. As fibras da piaçava vêm sendo comercializadas desde o período colonial, após o descobrimento do Brasil. Naquela época, as fibras eram procuradas por navegadores de várias nacionalidades para fabricação de cordas, as quais eram utilizadas como amarras nas embarcações (BARRETO, 2009), uma herança cultural atribuída aos índios das etnias Tupinambá e Tupiniquim, que residiam nas áreas de ocorrência natural da espécie.

A fibra vegetal da piaçava ocupa a $4^{\circ}$ posição entre os Produtos Florestais Não Madeireiros (PFNMs) brasileiros mais comercializados, com valor da produção estimada em
$\mathrm{R} \$ 103,72$ milhões de reais em 2016, correspondente a 45.645 toneladas $(45.645 .000 \mathrm{~kg})$, com decréscimo de $20,6 \%$ quando comparada aos valores de 2012 (57.462 toneladas), devido à baixa procura pelo produto. A erva-mate nativa (Ilex paraguariensis St. Hil., Aquifoleaceae), o açaí (Euterpe oleracea Mart., Arecaceae), as amêndoas do babaçu (Attalea speciosa Mart. ex. Spreng. Arecaceae), as fibras de piaçava (A. funifera), a castanha do Pará (Bertholletia excelsa Humb. \& Bonpl. Lecythidaceae) e o pó de carnaúba (Copernicia prunifera H. E. Moore. Arecaceae), juntos somaram $91,7 \%$ do valor total da produção extrativista vegetal não madeireira do Brasil. Entre esses PFNMs, apenas a fibra vegetal da piaçava (A. funifera) está relacionada ao Bioma Mata Atlântica. O buriti (Muritia flexuosa Mart., Arecaceae) e a carnaúba (C. prunifera) também fornecem fibras vegetais e, em 2016, foram comercializadas 
2.411 toneladas de carnaúba e 2.249 toneladas de buriti (IBGE, 2016; IPEA, 2015).

O sul do estado da Bahia é o maior produtor de fibras vegetais da piaçava, com destaque para os municípios de Ilhéus, Nilo Peçanha, Cairu, Ituberá e Canavieiras, os quais foram responsáveis por $92,80 \%$ da produção de piaçava nacional em 2016. Os vinte municípios produtores que compõe a zona de ocorrência natural da piaçava da Bahia $(A$. funifera) ocupam uma área com aproximadamente $57.380 \mathrm{~km}^{2}$ (Figura 1). A zona de ocorrência (no mapa em amarelo), segue até $60 \mathrm{Km}$ da linha do mar em direção ao interior do estado da Bahia, Brasil.

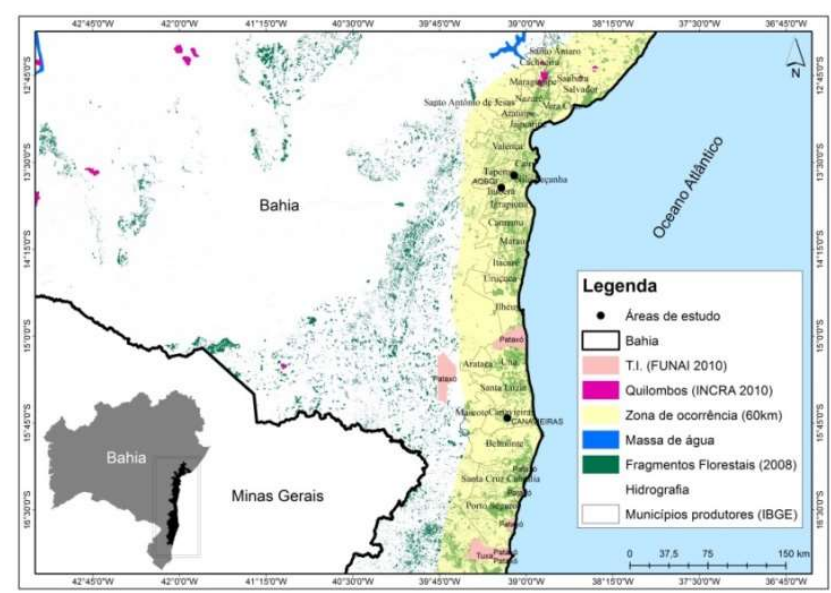

Figura 1. Zona de ocorrência natural da Attalea funifera, sul da Bahia.

Figure 1. Natural occurrence zone Attalea funifera, southern Bahia.

A fibra da piaçava da Bahia é resistente, rígida, lisa, de textura impermeável, variando de marrom claro à marromvermelho escuro e, na forma, desde cerca de $5 \mathrm{~mm}$ de diâmetro, na base, até a espessura de um fio de cabelo na extremidade superior, tendo em média $1,1 \mathrm{~mm}$ de espessura e alcançando 3,5 $\mathrm{m}$ de comprimento. Além disso, conservam sua elasticidade quando umedecidas (AQUINO, 2003). Fornari; Fornari-Junior (2013) afirmam que a fibra apresenta resistência ao desgaste e às intempéries, e seu baixo peso e elevado comprimento, propriciam a exploração máxima da sua propriedade de tração. As fibras limpas podem atingir mais de $2 \mathrm{~m}$ de comprimento, têm grande resistência à ruptura e, a elasticidade varia entre 1,75\% e 9\% (Guimarães, 2012).

A fibra da piaçava (A. funifera) possui elevada impermeabilidade e rigidez pois é rica em lignina $(48,4 \%)$, enquanto as fibras da juta (Corchorus capsularis Malvaceae) possuem teor de 16\% de lignina e o sisal (Agave sisalana Perrine, Agavaceae) possui $10 \%$ de lignina. Os teores de celulose correspondem a $31,6 \%$ nas fibras de piacava ( $A$. funifera) e 60\% de celulose na juta (C. capsulares) e no sisal (A. sisalana). A temperatura de inicio da decomposição térmica e degradação das fibras de piaçava foram de $225^{\circ} \mathrm{C}$, menor que a juta $\left(282^{\circ} \mathrm{C}\right)$ e o sisal $\left(302^{\circ} \mathrm{C}\right.$ ). Essas características fazem da fibra de piaçava um material promissor para sua utilização como reforço em compósitos poliméricos (Aquino, 2003).

O objetivo deste artigo é mensurar os rendimentos de fibra limpa, cortada, fino-média, grosso-média e subproduto gerado ("fita") do processsamento da fibra vegetal da piaçava da Bahia (A. funifera); quantificação total de resíduos formados; rendimento do conjunto de atividades e equipamentos necessários para o beneficiamento da fibra de piaçava; a partir da etapa de extração da fibra bruta até a confecção de vassouras.

A análise integrada do processamento dos PFNMs oriundos da fibra vegetal permite que os produtores conheçam as etapas das quais eles não participam, contribuindo para que os próprios atores locais, possam criar tecnologias sociais adequadas a realidade local e inovar no processo produtivo. A visão sistêmica permitiu mensurar o índice de rendimento e resíduos formados no processamento como um todo.

\section{MATERIAL E MÉTODOS}

Os ensaios de rendimento foram realizados em tres municipios da Bahia: Ituberá (13 44'15' S e 39 08' 47' W), Nilo Peçanha $\left(13^{\circ} 36^{\prime} 36^{\prime \prime} \mathrm{S}\right.$ e $\left.39^{\circ} 06^{\prime} 13^{\prime \prime} \mathrm{W}\right)$ e Canavieiras (15 46' $37^{\circ}$ ' S e $\left.38^{\circ} 58^{\prime} 19^{\prime \prime} \mathrm{W}\right)$. Dentro desses municípios, os seis sistemas produtivos analisados foram: a) sistema I corresponde à Associação Quilombola do Brejo Grande de Ituberá (AQBGI) localizada na zona rural de Ituberá, $35 \mathrm{~km}$ da sede do município, onde foram avaliadas as etapas de prébeneficiamento e beneficiamento da fibra vegetal da piaçava; b) sistema II - corresponde aos trabalhadores 'meeiros' que extraem em parceria com o produtor da Fazenda Nova Vida, localizada a $30 \mathrm{~km}$ da sede do munícipio de Canavieiras, onde foi avalida a etapa de pré-beneficiamento junto aos extrativistas 'meeiros'; c) sistema III - "catadouro familiar" localizado na zona rural a $25 \mathrm{~km}$ da sede do município de Nilo Peçanha, unidade produtiva que recebe a fibra vegetal bruta e comercializa a fibra cortada; d) sistema IV - "catadouro familiar" localizado na cidade de Ituberá, unidade produtiva onde é processada a fibra vegetal bruta e comercializa cortada, para a COOPRAP (Cooperativa das Produtoras e Produtores Rurais da APA - Area de Proteção Ambiental- do Pratigi); e) sistema V - COOPRAP, localizada na cidade de Nilo Peçanha, este sistema processa as fibras cortadas e realiza a confecção de vassouras de cepo com 18 furos cada, analises em março de 2014 (Va) e em outubro de 2012 (Vb); f) sistema VI - COOPAFBASUL (Cooperativa dos Agricultores Familiares do Baixo Sul), localizada na cidade de Ituberá, avaliou-se a confecção de vassouras de cepo com 18 furos cada.

A metodologia utilizada para avaliar o processo produtivo em cada um desses sistemas consistiu: (a) aplicação de questionário junto a duas cooperativas locais, COOPRAP e COOPAFBASUL; (b) visita in loco para acompanhamento da rotina produtiva nos "catadouros familiares" das regiões de Ituberá, Nilo Peçanha e Canavieiras; (c) realização de ensaios de rendimento em todos os seis sistemas produtivos.

Os equipamentos utilizados nos ensaios de rendimento foram os mesmos que os agroextrativistas e cooperativas utilizam em seu respectivo sistema produtivo. A única execessão foi a balança eletrônica modelo digital Prix 3/Prix 3 (bateria), com capacidade até $30 \mathrm{~kg}$, três casas decimais, que foi utilizada em todos os ensaios de rendimento.

O delineamento experimental para a análise integrada do processamento da fibra vegetal foi elaborado em conformidade com a organização produtiva de cada sistema, em consonância com as especificidades técnicas da etapa analisada e em quantidades diversas, conforme o produto 
processado. Portanto o número de repetições em cada ensaio de rendimento, a quantidade de massa de fibra por repetição e sistema produtivos foram diversas, conforme detalhado na
Tabela 1. Esses dados foram levantados in loco ao longo dos anos 2012, 2013 e 2014 (Tabela 1).

Tabela 1. Delineamento Experimental do Processamento dos PFNMs oriundos da fibra vegetal da Piaçava da Bahia. Table 1. Experimental design of NTFPs processing originating from the vegetable fiber piassava palm tree of Bahia.

\begin{tabular}{|c|c|c|c|c|c|c|}
\hline Sistema & $\mathrm{N}^{\circ}$ & $\mathrm{Rp}$ & $\mathrm{kg}$ fibra/sistema & $\mathrm{kg}$ total/sistema & Produto inicial & Mês/Ano \\
\hline Quilombo Brejo Grande & I & 14 & 4,0 & 56 & Bruta & $\operatorname{Jan} / 14$ \\
\hline Quilombo Brejo Grande & I & 8 & 2,0 & 16 & Limpa & Abril/13 \\
\hline 'Meeiros' Canavieiras & II & 8 & 4,0 & 32 & Bruta & Abril/13 \\
\hline “Catadouro” Alan & III & 5 & 7,0 & 35 & Bruta & Out/12 \\
\hline 'Catadouro' Hamilton & IV & 14 & 3,0 & 42 & Limpa & Março/14 \\
\hline COOPRAP & $\mathrm{Va}$ & 14 & 2,0 & 28 & Cortada & Março/14 \\
\hline COOPRAP & $\mathrm{Vb}$ & 5 & 2,6 & 13 & Cortada & Out/12 \\
\hline COOPAFBASUL & VI & 8 & 2,0 & 16 & Cortada & Abril/13 \\
\hline
\end{tabular}

Rp: número de repetições realizadas nos ensaios de rendimento.

As variáveis mensuradas nestes ensaios de rendimento foram: (I) pré-beneficiamento - tempo de separação (TS) da fibra e 'fita', massa de fibra bruta (MFB), massa da fibra vegetal limpa comercializável (MFL), massa de "fita" (MF) e massa de resíduo formado (MRF); (II) beneficiamento tempo de seleção e cata (TSC) das fibras limpas, massa da fibra cortada comercializável (MFC), massa de resíduo formado (MRF); (III) tempo de confecção das vassouras (TCV), massa de fibras fino-médias (MFF), massa de fibras grosso-médias (MFG) e, massa de resíduo formado (MRF) nesta etapa produtiva.

Os rendimentos foram determinados pela relação da massa do PFNM dividido pela massa da matéria prima original, para cada produto, em cada experimento e, entre experimentos (I ao VI), esses dados foram tratados através de análises estatísticas descritivas.

O rendimento do beneficiamento da fibra bruta vegetal até obtenção da fibra fino-médias, para confecção das vassouras de piaçava, ou seja, o rendimento do processo produtivo (RdProc) foi dado pela Equação 1, que considera os coeficientes encontrados em cada uma das etapas produtivas.

$\operatorname{RdProc}(\%)=(($ RdCata $) \times($ RdCorte $) \times($ RdFina $)) \times 100$

(Eq. 1)

em que: $\mathrm{RdCata}=$ rendimento de cata; $\mathrm{RdCorte}=$ rendimento de corte; RdFina= rendimento de fibra fino-média

Após a definição dos rendimentos dos PFNMs ajustou-se uma equação para calcular o índice do rendimento em massa da fibra vegetal fino-média da piaçava (MFP); rendimento em massa de "fita" (MF); rendimento em massa de fibra grossomédia (MFG); e rendimento em massa dos resíduos formados (MRF); todos em relação à massa de fibra bruta (MFB), ou seja, a matéria prima extraída da palmeira da piaçava. A renda mensal extrativista foi mensurada considerando os coeficientes técnicos aqui apresentados e informações de campo.

\section{RESULTADOS}

\subsection{PFNMs da piaçava da Bahia}

Os PFNMs oriundos da palmeira da piaçava da Bahia são: fibra vegetal bruta; fibra limpa; fibra cortada; vassouras de fibra vegetal; 'fitas' para cobertura de quiosques; artesanatos da fibra vegetal; material propagativo para plantio; biojóias do endocarpo dos frutos secos; óleo vegetal da semente dos frutos; polpa de 'satim'; artesanatos da folha (bolsas, esteiras, chapéus) e o palmito utilizado na alimentação tradicional quilombola e indígena (Figura 2).

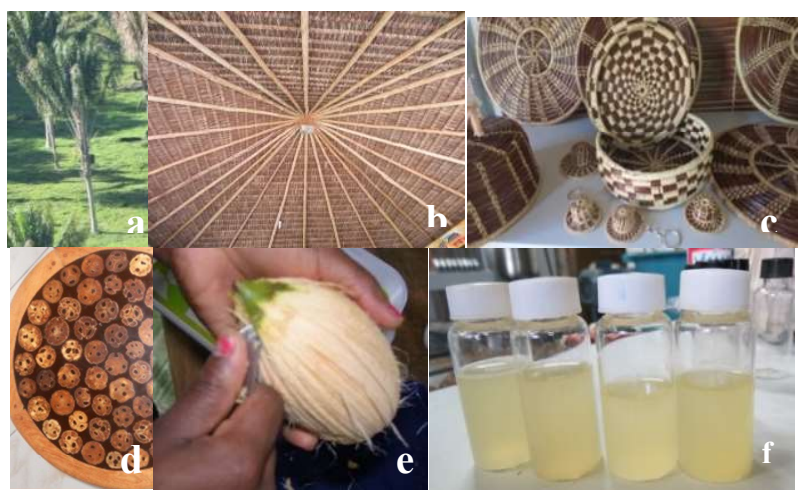

Figura 2. a) Palmeira piaçava; b) telhado da "fita"; c) artesanatos da fibra; d) artesantos de coco; e) polpa satin; e f) óleo vegetal das sementes.

Figure 2. a) Piassava palm tree; b) "stripe" roof; c) fibers crafts; d) coconut crafts; e) "satin" pulp; and f) vegetable oil.

Em termos de qualidade a fibra vegetal é considerada de primeira (tipo exportação) quando apresenta a cor avermelhada, tem uma espessura fino-média, adequada para vassouras de cepo, de chita e chapa de uso doméstico, entre outras finalidades. Em Canavieiras e Ituberá, os informantes foram precisos ao afirmar que essas fibras vegetais de primeira são encontradas em palmeiras que sofrem extrações, em intervalos, entre 08 e 12 meses, palmeiras em estagio inicial de desenvolvimento, bem como em determinadas regiões como Canavieiras, Belmonte e Nazaré, fato que sugere um variabilidade morfológica nas plantas dessas áreas. O mercado consumidor exige que as fibras tipo exportação estejam secas, sem impurezas e bem penteadas (soltas), inteiras ou cortadas em tamanhos específicos.

As fibras de segunda apresentam uma cor marrom claro ou escuro, possuem espessura entre fino-média e grossomédia, são destinadas à confecção de vassouras redondas (vassouras tradicionais), vassouras de gari e vassourões. As fibras grosso-médias são menos flexíveis do que as de fibras de primeira e, portanto menos trabalháveis.

Outra diferença entre as fibras de primeira e de segunda está relacionada com o tamanho dos fios de fibras limpas, comprimento original (extração), pois os feixes de piaçava são classificados, como sendo: fibras de primeira, aquelas com comprimento $\geq 1,20 \mathrm{~m}$; e de segunda, feixes de fibras limpas 
entre 0,5 a 1,0 m de comprimento. Existe ainda a fibra curta, com comprimento de até $0,5 \mathrm{~m}$, localmente denominadas de "tocos".

\subsection{Fibra vegetal bruta}

A fibra vegetal bruta é o PFNM extraído a partir das palmeiras com aproximadamente dez anos de idade, quando a planta entra na fase de desenvolvimento denominada de "bananeira em produção", momento em que se pode iniciar a exploração. Respeitando-se as boas práticas de manejo as plantas fornecem fibras brutas (Figura 3), até a morte da palmeira, já na fase "coqueiro".

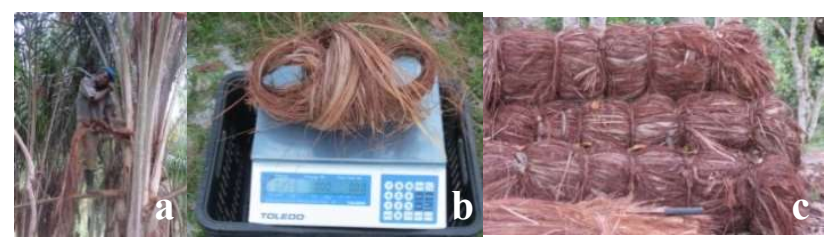

Figura 3. a) Extração da fibra bruta; b) pesagem; e c) armazenamento ("mondongos") de fibra bruta.

Figure 3. a) Extraction of raw fiber; b) weighing; and c) storage ("mondongos") of raw fiber.

Esse produto é extraído e comercializado no sul da Bahia, entre agroextrativistas, produtores e atravessadores que fazem o transporte até as fábricas de vassouras nos estados do Rio de Janeiro, São Paulo, Espírito Santo e Minas Gerais.

Segundo IBGE (2013) o valor total comercializado de fibras brutas vegetais, no estado da Bahia, foi de $\mathrm{R} \$$ 80.003.000,00 (US\$ 34.151.370,27), portanto, o preço médio comercializado da fibra vegetal bruta foi de $\mathrm{R} \$ 1,88 / \mathrm{kg}$ (US\$ $0,80 / \mathrm{kg}$ ) em 2013. Entretanto os valores pagos aos extrativistas para realizarem a extração da fibra bruta são inferiores a este valor médio apontado pelo IBGE. Em 2014 o valor pago ao extrator da fibra bruta, variou variam entre $\mathrm{R} \$ 0,73 / \mathrm{kg}$ (US\$ 0,73/kg) a R $\$ 2,00 / \mathrm{kg}(\mathrm{US} \$ 0,85)$. Diante desta conjuntura os extrativistas acessaram a Política de Garantia de Preços Mínimos para os Produtos da Sociobiodiversidade (PGPM-Bio), entre anos de 2010 a 2013. Em 2013, o preço mínimo, pago aos extrativistas, estipulado pelo governo federal, foi de R $\$ 1,67 / \mathrm{kg}$ (US\$ 0,71/kg).

Considerando-se o valor médio de R $\$ 0,80 / \mathrm{kg}$ (US\$ 0,34$)$ de fibra bruta extraída, pago ao extrativista, em 2014, bem como a sua produtividade média de $37,5 \mathrm{~kg}$ de fibra bruta por dia e por pessoa, estimou-se uma renda mensal média de aproximadamente $\mathrm{R} \$ 600,00 /$ mês (US $\$ 255,32 /$ mês).

\subsection{Fibra vegetal limpa}

A fibra vegetal limpa é o PFNM resultante da etapa de pré-beneficiamento da fibra vegetal bruta, que também gera o subproduto conhecido como "fitas", além dos resíduos formados com material fora dos padrões requeridos pelo mercado. A atividade consiste na separação manual das fibras e "fitas", com auxilio do facão, "escova" e "pente metálico" (Figura 4).

O pré-beneficiamento da fibra vegetal, localmente é denominado de "cata" da fibra vegetal bruta, etapa genuinamente feminina onde as extrativistas são conhecidas por "catadeiras". Essas extrativistas trabalham em "catadouros familiares".

Os ensaios de rendimento realizados em Canavieiras, Ituberá e Nilo Peçanha, apontaram para um rendimento médio de 65,6\% em fibras limpas; formação de 17,1\% de "fitas" e 17,3\% de resíduos (Tabela 2).

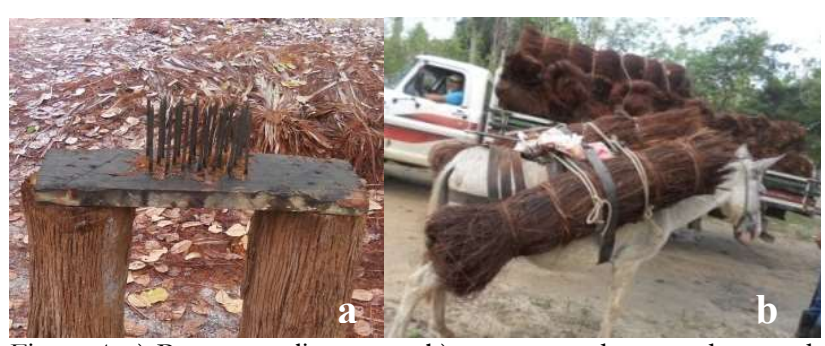

Figura 4. a) Pente para limpeza; e b) transporte dos mondongos de fibra vegetal limpa.

Figure 4. a) Comb for cleaning; and b) transportation of clean vegetable fiber.

Tabela 2. Rendimento em fibra limpa, "fitas" e resíduo do prébeneficiamento.

Table 2. Yield in clean fiber, "stripes" and waste pre- beneficiation.

\begin{tabular}{ccccccc}
\hline \multirow{2}{*}{ Sist. } & Mi & \multicolumn{2}{c}{ Fibra (\%) } & \multicolumn{2}{c}{ Fita (\%) } & Resíduo \\
\cline { 3 - 7 } & $(\mathrm{Kg})$ & Rend. & C.V. & Rend. & C.V & Rend \\
\hline I & 4,0 & 67,2 & 9,1 & 15,1 & 25,9 & 17,7 \\
II & 4,0 & 63,5 & 8,2 & 20,4 & 15,6 & 16 \\
III & 7,0 & 66,0 & 6,2 & 15,9 & 13,2 & 18,1 \\
\hline \multicolumn{2}{c}{ Médias } & 65,6 & 7,9 & 17,1 & 18,1 & 17,3 \\
\hline
\end{tabular}

Mi: Massa inicial.

A maior formação de resíduo está relacionada a uma limpeza e seleção mais apurada, conforme exigências dos atravessadores. Por outro lado, a menor formação de resíduos, nesta etapa, está relacionada à estratégia dos extrativistas elevarem seus ganhos por produção de fibra limpa gerada ("meeiros").

O tempo mensurado nos ensaios de rendimentos demonstra a agilidade da "catadeira" na separação entre fibra e "fita". A média de tempo gasto na "cata" da piaçava é de aproximadamente 5,9 min para transformar $1 \mathrm{~kg}$ de fibras brutas em fibras limpas e "fitas". Então a produtividade média diária de uma "catadeira" é de aproximadamente $80 \mathrm{~kg}$ de fibra vegetal bruta/dia/pessoa.

As fibras limpas são comercializadas no mercado local, em média, por US\$ 0,98/ kg ( R \$ 2,30/Kg). As extrativistas que fazem a separação e limpeza recebem por produção, aproximadamente US\$0,23/ $\mathrm{kg}(\mathrm{R} \$ 0,53 / \mathrm{kg})$ de fibras limpas. Considerando os coeficientes de rendimento em fibras limpas, preços médios e a produtividade por pessoa, a renda mensal da extrativista fica em torno de US\$240.89 (R\$ $566,08)$ para pré-beneficiar $1.618 \mathrm{~kg}$ de fibras vegetais brutas

\subsection{Fibra vegetal cortada}

A fibra vegetal cortada é o PFNM da piaçava tipo exportação, obtida após o beneficiamento da fibra limpa da piaçava. Essa etapa consiste na reabertura dos fardos limpos de piaçava, nova seleção das fibras, conforme sua espessura e comprimento e o descarte dos resíduos e fibras grossas.

Os equipamentos utilizados na etapa de beneficiamento são os "pentes" para limpezas e ajustes nas direções das fibras, a guilhotina para cortar as fibras nos comprimentos requeridos, facão, machadinha, balança e pequenas ferramentas para manutenção e ajustes dos equipamentos. Os cortes variam entre $37 \mathrm{~cm} ; 32 \mathrm{~cm} ; 30 \mathrm{~cm} ; 24 \mathrm{~cm} ; 20 \mathrm{~cm} ; 15$ $\mathrm{cm}$; e $13 \mathrm{~cm}$ de comprimento.

As fibras são selecionadas e separadas entre fino-médias e grosso-médias (comercializáveis) e grossas (não 
comercializáveis), as duas primeiras espessuras são aproveitadas, no Brasil, para confecção das vassouras de piaçava, as fibras grossas são descartadas, pois, se utilizadas, rompem ao serem dobradas e fixadas no cepo da vassoura.

Considerando os três sistemas produtivos analisados, os ensaios de rendimento apontaram uma média aproximada de $73,2 \%$ de fibras limpas e cortadas; e a formação de aproximadamente 26,8\% resíduos na etapa de beneficiamento da fibra vegetal da piaçava, conforme Tabela 3.

Tabela 3. Rendimento em fibra vegetal cortada e resíduo do beneficiamento.

Table 3. Yield on cut vegetable fiber and residue beneficiation.

\begin{tabular}{ccccc}
\hline \multirow{2}{*}{ Sist. } & \multirow{2}{*}{ Mi $(\mathrm{Kg})$} & \multicolumn{2}{c}{ FIBRAS $(\%)$} & RESÍD. $(\%)$ \\
\cline { 3 - 5 } & & Rend. & C.V. & Rend. \\
\hline I & 2,0 & 61,3 & 12,4 & 38,7 \\
III & 4,7 & 89,5 & 10,8 & 10,5 \\
IV & 3,0 & 68,9 & 68,9 & 31,1 \\
\hline \multicolumn{2}{c}{ Médias } & 73,2 & 30,7 & 26,8 \\
\hline
\end{tabular}

Mi: Massa inicial.

Quanto mais rígido o critério de aproveitamento das fibra vegetais menor o rendimento em fibras vegetais, e maior a formação de resíduos, como ocorreu no sistema I (AQBGI). Por outro lado, sistemas produtivos que fornecem aos atravessadores, que buscam pagar menores preços pelo produto, adquirem fardos de fibras cortadas com espessuras indesejadas, bem como a presença de outras impurezas.

Os agroextrativistas e produtores reúnem em um mesmo lote (amostra) feixes de fibras limpas, oriundos de diferentes áreas de extração, bem como de diferentes palmeiras, em diversas fases de desenvolvimento. Fato comum na atividade florestal, onde os produtos vegetais, oriundos das florestas nativas, são heterogêneos, o que exige sistemas produtivos que abarque as especificidades do PFNM.

O tempo de corte de $1 \mathrm{~kg}$ de fibra vegetal limpa é de 11,2 minutos, ou seja, em 2 horas e 48 minutos o extrativista beneficia $15 \mathrm{~kg}$ de fibra limpa. Acrescentando o tempo de preparo para o corte (12 minutos $/ 15 \mathrm{~kg}$ ), a capacidade produtiva do extrativista é de beneficiar (cortar) aproximadamente duas arrobas e meia ( $37 \mathrm{~kg} / \mathrm{dia} /$ pessoa) de fibra vegetal da piaçava por dia de trabalho.

Os extrativistas que realizam essa etapa de beneficiamento e, trabalham por produção, recebem aproximadamente US\$ $0,55 / \mathrm{kg}(\mathrm{R} \$ 1,30 / \mathrm{kg})$ de fibras cortadas, o equivalente a uma renda mensal de aproximadamente US\$314,98 (R \$ 740,20).

No mercado local as fibras cortadas são comercializadas em média por US\$2,99/kg (R\$4,67/ kg). A renda líquida mensal do extrativista que é dono do "catadouro" pode chegar no máximo US\$369,60 (R \$ 868,56) para beneficiar $740 \mathrm{~kg}$ de fibra vegetal limpa, caso encontre mercado para escoar sua produção.

\subsection{Vassoura de fibra vegetal}

As fibras cortadas sofrem novos processamentos na confecção das vassouras da piaçava (Figura 5). Os feixes de fibras cortadas são abertos e o operador inicia a classificação das fibras em fibra fino-média, grosso-média e o descarte de fibras vegetais fora do padrão, formando novos resíduos.

As fibras grosso-médias são utilizadas nos vassourões de limpeza urbana, vassouras de chapa e redondas (tradicional). Essas fibras grosso-médias representam 11,5\% do total de fibras cortadas adquiridas para confecção das vassouras de piaçava.

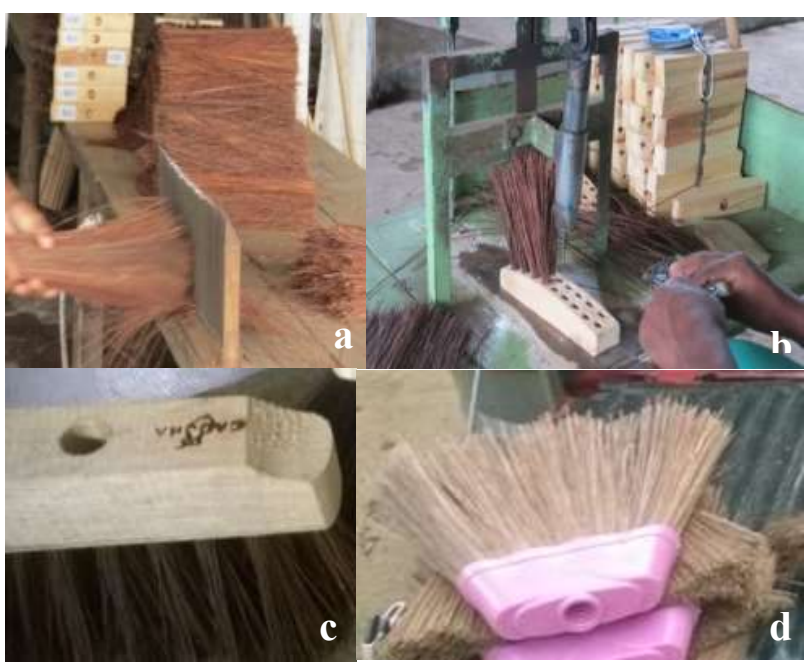

Figura 5. a) Seleçã e limpeza; b) grampeamento da fibra vegetal limpa; c) vassouras de cepo; e d) vassoura de chapa.

Figura 5. a) Selection and cleaning; b) stapling of clean vegetable fiber;c) brooms "stump"; and d) brooms sheet.

As fibras vegetais de espessura fino-médias são destinadas a confecção das vassouras de cepo de madeira (uso doméstico), entre outros modelos de vassouras. Este PFNM oriundo da piaçava da Bahia é o mais procurado e valorizado. O rendimento médio de fibras fino-médias, considerando os três sistemas avaliados, foi de $74 \%$ de aproveitamento de fibras vegetais cortadas, e gerou um resíduo de aproximadamente $15,3 \%$ na etapa de confecção de vassouras de cepo (uso doméstico) conforme Tabela 4.

Tabela 4. Rendimentos em fibra fino-média, grosso-média e resíduo na etapa de confecção da vassoura de cepo.

Table 4. Yields in thin-medium fiber, thick-medium fiber and residue in the manufacturing stage of the stump's broom.

\begin{tabular}{lcccc}
\hline \multirow{2}{*}{ Sist } & \multirow{2}{*}{ Mi $(\mathrm{kg})$} & \multicolumn{3}{c}{ Rendimento (\%) } \\
\cline { 3 - 5 } & & Fibra fina-média & Fibra grossa & Resíduo \\
\hline $\mathrm{Va}$ & 2,0 & 76 & 11 & 10 \\
$\mathrm{Vb}$ & 3,0 & 71 & 12 & 13 \\
$\mathrm{VI}$ & 1,2 & 75 & - & 23 \\
\hline & Médias & 74 & 11,5 & 15,3 \\
\hline
\end{tabular}

Mi: Massa inicial.

Os 'tufos' ou maços de fibras fino-médias que são fixados no cepo da vassoura, com auxílio de um equipamento denominado de grampeadora ("pica pau"), manualmente, cada 'tufo' equivale em média a 7,75 gramas/furo nas vassouras de cepo com 18 furos. Portanto cada vassoura de cepo de madeira com 18 furos, necessita de aproximadamente $140 \mathrm{~g}$ de fibras fino-médias de piaçava para sua confecção. Esse valor mensurado ficou próximo ao que a COOPRAP adotava nas suas análises do custo de produção, ou seja, 164 $g$ por vassoura de cepo tradicional.

A produtividade média de cada operador, considerando uma hora de trabalho, é de 9,5 vassouras de cepo com 18 furos. Considerando que $1 \mathrm{~kg}$ de fibra fino-média é possível confeccionar 6,22 vassouras, a produtividade diária dos operadores foi de 72 vassouras de cepo por dia. Deve-se considerar mais um operador para apoio na consolidação 
dessas 72 vassouras, ou seja, a produtividade de um operador é de 36 vassouras por dia.

\subsection{Rendimento do processo produtivo}

Neste estudo, identificou-se que aproximadamente 41,8\% de todo o material vegetal processados, transformaram-se em resíduos; 35,7\% foram transformados em fibras fino-médias, produto utilizado na confecção de vassouras de chita doméstica; $17 \%$ dessa massa corresponderam às "fitas" ou "pentes para cobertura de quiosque" e 5,3\% correspondem às fibras grosso-médias, utilizadas nas vassouras tradicionais (redondas) e vassouras de limpeza urbana (Tabela 5).

Tabela 5. Rendimento total do processamento da fibra vegetal bruta em fibras vegetais fino-médias.

Table 5. Total yield of crude fiber into vegetable thin-medium fibers.

\begin{tabular}{lcccc}
\hline \multirow{2}{*}{ PFNM } & \multicolumn{4}{c}{ Rendimento do processamento (\%) } \\
\cline { 2 - 5 } & $\begin{array}{c}\text { Pré- } \\
\text { beneficiamento }\end{array}$ & Beneficiamento & $\begin{array}{c}\text { Confecção } \\
\text { vassoura }\end{array}$ & Total \\
\hline $\begin{array}{l}\text { Fibra fina- } \\
\text { média }\end{array}$ & 65,6 & 73,2 & 74,0 & 35,6 \\
$\begin{array}{l}\text { Fibra grosso- } \\
\text { média }\end{array}$ & - & - & 11,5 & 5,3 \\
Fitas & 17 & - & - & 17 \\
Resíduo & 17,3 & 26,8 & 15,3 & 41,8 \\
\hline
\end{tabular}

Através do índice de rendimento apresentado na Tabela 5 podemos obter a massa fibra vegetal fino-média (MFF) geradas a partir de uma quantidade de massa vegetal bruta (MFB) conhecida, conforme Equação 2.

$$
\mathrm{MFF}=\mathrm{MFB} \times 0,36
$$

em que: $\mathrm{MFF}=$ massa de fibra vegetal fino-média (kg); MFB = massa de fibra vegetal bruta $(\mathrm{kg})$.

Considerando que as fibras grosso-médias também são aproveitadas na confecção das vassouras de limpeza urbana, vassouras de chapa e vassouras tradicionais (redondas), a massa de fibras grosso-médias (MFG) pode ser obtida pela Equação 3.

$$
\mathrm{MFG}=\mathrm{MFB} \times 0,05
$$

em que: $\mathrm{MFG}=$ massa de fibra vegetal grosso-média (kg); MFB = massa de fibra vegetal bruta $(\mathrm{kg})$.

As "fitas" também são comercializadas no mercado local, regional e nacional, subproduto aproveitado para construção e coberturas de quiosques, segue a Equação 4 para calcular a massa "fita" (MF):

$$
\mathrm{MF}=\mathrm{MFB} \times 0,17
$$

em que: $M F=$ massa de "fita" $(\mathrm{kg}) ; \mathrm{MFB}=$ massa de fibra vegetal bruta inicial $(\mathrm{kg})$.

\section{DISCUSSÃO}

O presente estudo apresenta uma compilação de informações que estavam dispersas e, portanto, ainda não apresentadas aos produtores e comunidade científica. Empregou-se uma metodologia adequada a realidade produtiva local, onde os ensaios de rendimentos foram padronizados nos diferentes sistemas produtivos, compondo com procedimentos específicos para cada etapa da cadeia produtiva. Diante deste contexto, a discussão dos resultados encontrados neste estudo em relação aos estudos realizados pelos diversos autores (MOREAU, 1997; SAVASTANO JR.; PIMENTEL, 2000; D'ALMEIDA ET AL., 2006; AQUINO, 2003), informações repassadas pelas unidades produtivas das associações, cooperativas e produtores locais da fibra vegetal, devem ser analisados com cautela.

Moreau (1997) estimou que o percentual de desperdício nas indústrias, que trabalham com esta fibra, é da ordem de $20 \%$. Esta estimativa é inferior ao valor apresentado neste estudo para a etapa de beneficiamento (limpeza e corte da fibra vegetal), onde $26,8 \%$ do material vegetal é considerado resíduo florestal. A transformação total da fibra vegetal em vassouras de cepo gera $41,8 \%$ de resíduo florestal, fato que aponta para a necessidade de pesquisa quanto ao aproveitamento deste subproduto.

Savastano Jr.; Pimentel (2000) afirmaram que na etapa de limpeza e penteamento (pré-beneficiamento) das fibras vegetais, perde-se cerca de $30 \%$ do material e, que estes são queimados ao ar livre. Além dos materiais que são descartados na etapa de confecção das vassouras. Apesar do estudo acima não diferenciar com clareza as etapas de pré-beneficiamento e beneficiamento, conforme exposto neste trabalho, os autores esclarecem que não se trata da etapa de corte da fibra vegetal (beneficiamento), portanto o valor apresentado por Savastano Jr.; Pimentel (2000) esta acima dos valores calculados nos ensaios de rendimento para etapa de prébenefiicamento (limpeza e penteamento), ou seja, nesta etapa forma-se $17,3 \%$ de resíduo e $17 \%$ de "fitas" para cobertura de quiosque.

A estimativa de perdas no processamento da fibra vegetal da piaçava nas industrias de transformação, após a produção das vassouras de piaçava, foi estimada em 30\% (D'Almeida et al., 2006; Aquino, 2003). As unidades produtivas analisadas neste estudo, apresentaram perdas na ordem de 15,3\% na etapa de confecção das vassouras, isoladamente.

A COOPRAP estimou que o rendimento do processo produtivo, no ano de 2013 , da etapa de transformação da fibra bruta até a confecção da vassoura de cepo, estava em torno dos $37,33 \%$, valor inferior ao encontrado nesta pesquisa, onde a formaçao de resíduo da etapa de prébeneficiamento até a confecção das vassouras de cepo foi estimado na ordem de $41,8 \%$.

Os coeficientes técnicos apresentados pela COOPAFBASUL, em 2014, com relação aos rendimentos do processo produtivo e, formação de resíduos nos três galpões foram: etapa de pré-beneficiamento apresenta $10 \%$ de resíduo, $20 \%$ de 'fita' e $70 \%$ de fibra limpa; etapa de beneficiamento forma-se $12 \%$ de resíduo. Esses valores também divergem dos encontrados neste estudo, principalmente quanto aos resíduos formados. Acredita-se que essas diferenças encontradas são decorrente da utilização das fibras grosso-média, misturadas com as fibras fino-média na confecção de vassouras de cepo ou de chita. Bem como pelo fato da produção de vassouras tradicionais (redondas), estas permitem a utilização das fibras grosso-média. Os valores de rendimento de "fitas" estão próximos ao apontado por este estudo, $17 \%$ de "fitas". A quantidade de fibras finomédia ou fibra limpa, como a cooperativa classifica, foram bem similares.

Conforme os resultados apresentados, detaca-se que a confecção de vassouras foi a etapa que a menor formação de 
resíduo, seguida da etapa de pré-beneficiamento (limpeza e penteamento). A etapa de beneficiamento (corte da fibra vegetal), apresentou a maior geração de resíduos em comparação às demais etapas do processamento da fibra vegetal. Isso ocorre, pois, a fibra vegetal é um produto heterogêneo e, nesta etapa produtiva, são realizadas rígidas seleções afim de contemplar as exigências do mercado, principalmente o mercado externo.

Com base nas informações expostas é possível apresentar um cenário de vertilização do processamento, onde o agroextrativista realiza todas as etapas do processo produtivo, ao longo de um mês. Admite-se, para tanto, que as unidades produtivas familiares ("catadouros familiares") estejam equipadas, funcionando e escoando a sua produção.

A média de produtividade diária de um único extrativista é de aproximadamente $37,5 \mathrm{~kg}$ de fibra vegetal bruta, após uma semana de trabalho, ou cinco dias de trabalho, ele terá extraído e transportado até o "catadouro familiar", o total de $187,5 \mathrm{~kg}$ (12,5 arrobas) de fibras brutas. Após o prébeneficiamento da fibra vegetal bruta, o extrativista terá $121,88 \mathrm{~kg}(8,13$ arrobas $)$ de fibras limpas e 31,88 kg (2,13 arrobas) de "fitas". As fibras limpas são comercializadas no mercado local por R $\$ 31,00 / 15 \mathrm{~kg}$ (US\$ 13,23/15kg) e R $\$$ $18,50 / 15 \mathrm{~kg}$ (US\$ 7,90/15 kg) de "fitas".

Esses $121,88 \mathrm{~kg}$ de fibras limpas, após o corte/limpeza/seleção das fibras vegetais, é transformado em $88,97 \mathrm{~kg}(5,93$ arrobas $)$ de fibras cortadas com $37 \mathrm{~cm}$ de comprimento. Neste caso há formação de $32,91 \mathrm{~kg} \mathrm{em}$ resíduos de corte da fibra vegetal da piaçava. As fibras cortadas (beneficiadas) são comercializadas localmente por $\mathrm{R} \$ 4,67 / \mathrm{kg}$ (US\$1,99/kg) de fibras cortadas ou R \$ 70,00/15 $\mathrm{kg}$ (US\$29,88/15 kg) de fibra cortada.

Para confecção das vassouras de cepo de piaçava com 18 furos é necessário mais limpezas e seleções desses $88,97 \mathrm{~kg}$ de fibras cortadas, essa massa é decomposta em 65,84 kg (4,39 arrobas) de fibras fino-médias, além de $9,79 \mathrm{~kg}$ de fibras grosso-médias e 13,35 kg de resíduos. Com essa massa de fibras fino-médias é possível confeccionar 410 vassouras de cepo com 18 furos. Essas vassouras são comercializadas ao consumidor final por R $\$ 6,00$ (US\$2,56) cada, podendo gerar um ganho bruto de R\$2.460,00 (US\$ 1.050,12) por extrativista por mês, somente com as vassouras de piaçava.

O tempo de serviço do extrativista foi estimado em cinco dias para extrair, cinco dias para a atividade de prébeneficiamento deste volume de fibras brutas, três dias para cortar o volume gerado e mais doze dias para confeccionar as 410 vassouras de piaçava. Totalizando, no máximo, 25 dias para extrair as fibras, processar e produzir as vassouras, a partir das fibras brutas coletadas das palmeiras da piaçava.

Adotando-se os custos com transporte da Companhia Nacional de Abastecimento - CONAB (comunicação pessoal, 2013), de aproximadamente R $\$ 1,31$ (US\$ 0,56) por arroba, o extrativista transporta $187,50 \mathrm{~kg}$ de fibras brutas, da mata até o "catadouro familiar", então o custo com transporte equivale $\mathrm{R} \$ 16,38$ (US\$ 6,99). Os insumos necessários para produção de vassoura são: cepos de madeira, cabos, grampos, embalagens e rótulos. Estes insumos estimados pela COOPRAP em R \$ 1,90 (US\$ 0,81) por vassouras de cepo de madeira com 18 furos. Totalizando um custo de produção de $\mathbf{R} \$ 795,3$ (US $\$ 339,49$ ) para processar e transformar 187,50 kg (12,5 arrobas) de fibras brutas em 410 vassouras de piaçava.
Deste modo, subtraindo os ganhos brutos menos o custo de produção das 410 vassouras, o extrativista poderia auferir uma renda liquida mensal de aproximadamente $\mathrm{R} \$ 1.664,63$ (US\$ 710,59), trabalhando sozinho, durante os 25 dias no mês e no máximo oito horas por dia. Diariamente o agroextrativista receberia $\mathrm{R} \$ 66,57 / \mathrm{dia}$ (US\$ 28,42/dia).

\section{CONCLUSÕES}

A análise integrada do processamento da fibra vegetal da piaçava aponta para a necessidade da verticalização da cadeia produtiva, principalmente por parte dos extrativistas tradicionais, os quais devem se apropriar das técnicas e tecnologias envolvidas no processamento dos PFNMs oriundos dessa fibra vegetal.

Em escala local, dentro dos "catadouros familiares", os volumes de fibras manejados seriam menores com a verticalização da cadeia produtiva, melhorando a qualidade do trabalho manual, diminuindo os esforços individuais, elevando-se o aproveitamento da matéria-prima extraída e, diminuindo-se as perdas ao longo do processamento.

A diversidade e complexidade desta cadeia produtiva, pulveriza ganhos e eleva o volume (quantidade) de fibras vegetais manejadas, para auferir ganhos menores, quando comparado aos ganhos com a comercialização de produtos com valores agregados. Portanto a verticalização da produção dos PFNMs oriundos da fibra vegetal da piaçava, caso adotada e, praticada pelas comunidades tradicionais, provocaria uma menor pressão sobre às áreas de Mata Atlântica, onde a espécie cresce naturalmente, bem como diminuiria a demanda para formação de novos plantios.

O modelo de gestão dos sistemas produtivos analisados ainda é semelhante ao modelo adotado pelos portugueses na colonização, os capitães da época compravam a fibra bruta in natura dos extrativistas, vendiam e, auferiam melhores ganhos do que os extratores. Atualmente os agroextrativistas, quilombolas, atravessadores e "catadouros familiares", ainda prezam pela especialização das atividades de processamento, bem como tentam substituir a figura dos capitães, senhores de engenho e os atravessadores atuais, resistindo à verticalização da cadeia produtiva e a gestão total por parte dos extrativistas tradicionais.

\section{REFERÊNCIAS}

AQUINO, R. C. P. Desenvolvimento de compósitos de fibras de piaçava da espécie Attalea funifera Mart e matriz de resina poliéster. 151f. 2003. Tese (Doutorado) - Centro de Ciência e Tecnologia, Universidade Estadual do Norte Fluminense Darcy Ribeiro, Rio de Janeiro, 2003.

BARRETO, R. O. Técnicas de Manejo e sustentabilidade da palmeira Attalea funifera Martius - piaçava da Bahia. Candombá - Revista Virtual, Salvador, v. 5, n. 2, p. 8097, 2009.

D'ALMEIDA, J. R. M.; AQUINO, R. C. M. P.; MONTEIRO, S. N. Tensile mechanical properties, morphological aspects and chemical characterization of piassava (Attalea funifera) fibrs. Composites Part A: Applied Science and Manufacturing, Kidlington, v. 37, n. $9, \quad$ p. 1473-1479. DOI: https://dx.doi.org/10.1016/j.compositesa.2005.03.035 
GUimarÃES, C. A. L.; SILVA, L. A. M. Piaçava da Bahia (Attalea funifera Martius): do extrativismo à cultura agrícola. Ilhéus: Editus; 2012. 264 p.

IPEA_INSTITUTO DE PESQUISA ECONÔMICA APLICADA. Texto para Discussão $\mathbf{n}^{\circ} 2104$. Operacionalização da Política de Grantia de Preços Minimos para Produtos da Sociobiodiversidade 20092013: Há Espaço para Crescer. Viana, J. P. Instituto de Pesquisa Econômica e Aplicada, Brasilia, 2015. 28p.

IBGE_INSTITUTO BRASILEIRO DE GEOGRAFIA E ESTATÍSTICA. Produção da Extração Vegetal e Silvicultura. Rio de Janeiro: Instituto Brasileiro de Geografia e Estatistica. Acesso em outubro de 2017, disponível em: $<$ www.ibge.gov.br/home/estatistica/economia/pevs/20 $13>$.

FORNARI, C. I.; FORNARI JR, C. C. M. Avaliação da força de cisalhamento das fibras longas de piaçava em poliester insaturado. Estudos tecnológicos em Engenharia, São Leopoldo, v. 9, n. 1, p. 27-36, 2013. DOI: http://dx.doi.org/10.4013/ete.2013.91.04

MOREAU, M. S. Ocorrência, Produtividade e canais de Comercialização da Piaçava (Attalea funifera Mart.) em Ilhéus, Una e Canavieiras - Bahia. 64f. 1997. Dissertação (Mestrado) - Escola de Agronomia, Universidade Federal da Bahia, Cruz das Almas, 1997.

SAVASTANO, J. R. H.; PIMENTEL, L. L. Viabilidade do aproveitamento de reíduos de fibras vegetais para fins de obtenção de material de construção. Revista Brasileira de Engenharia Agricola e Ambiental, Campina 144 Grande, v. 4, p. 103-110, 2000. DOI: 